Math. Model. Nat. Phenom.

Vol. 5, No. 2, 2010, pp. 125-145

DOI: $10.1051 / \mathrm{mmnp} / 20105205$

\title{
Indecision in Neural Decision Making Models
}

\author{
J. Milton ${ }^{1 *}$, P. Naik ${ }^{2}$, C. Chan ${ }^{3}$ and S. A. Campbell ${ }^{4}$ \\ 1 Joint Science Department, The Claremont Colleges, Claremont, CA 91711, USA \\ ${ }^{2}$ Pomona College, The Claremont Colleges, Claremont, CA 91711, USA \\ ${ }^{3}$ Harvey Mudd College, The Claremont Colleges, Claremont, CA 91711, USA \\ ${ }^{4}$ Department of Applied Mathematics, University of Waterloo, Canada
}

\begin{abstract}
Computational models for human decision making are typically based on the properties of bistable dynamical systems where each attractor represents a different decision. A limitation of these models is that they do not readily account for the fragilities of human decision making, such as "choking under pressure", indecisiveness and the role of past experiences on current decision making. Here we examine the dynamics of a model of two interacting neural populations with mutual time-delayed inhibition. When the input to each population is sufficiently high, there is bistability and the dynamics is determined by the relationship of the initial function to the separatrix (the stable manifold of a saddle point) that separates the basins of attraction of two co-existing attractors. The consequences for decision making include long periods of indecisiveness in which trajectories are confined in the neighborhood of the separatrix and wrong decision making, particularly when the effects of past history and irrelevant information ("noise") are included. Since the effects of delay, past history and noise on bistable dynamical systems are generic, we anticipate that similar phenomena will arise in the setting of other physical, chemical and neural time-delayed systems which exhibit bistability.
\end{abstract}

Key words: neural network, decision, inhibitory, time delay, bistability AMS subject classification: 92C20, 34K20, 34K50

\footnotetext{
${ }^{*}$ Corresponding author. E-mail: jmilton@jsd.claremont.edu
} 

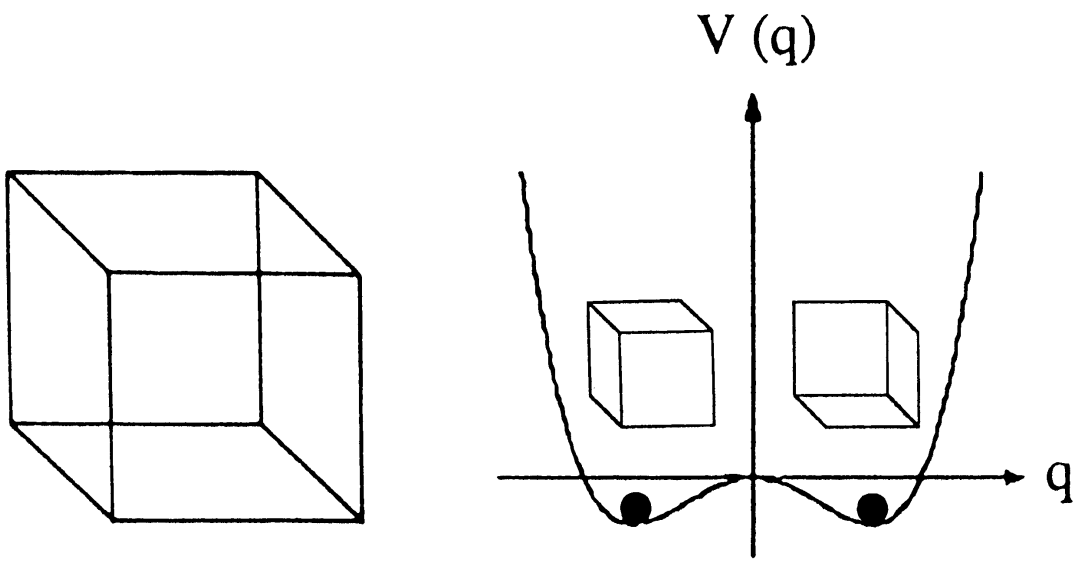

Figure 1: Schematic representation of the potential, $V(q)$, (right) for an ambiguous figure, the Necker cube (left). The two different perceptions of the Necker cube each correspond to a different well of the potential.

\section{Introduction}

An organism unable to make decisions is doomed forever to live at a crossroad. Thus it is not surprising that even the lowest organisms, e.g. bacteria [10] and invertebrates [7], have some form of decision making mechanism. A central element of contemporary cognitive models of human decision making is the notion that every determined choice is one that corresponds to the winner of a group of competing neuronal populations, each representing a different decision $[5,8,32,53,56]$. Multimodal imaging techniques indicate that human decision making involves a spatially distributed neural network [27, 38, 45, 47, 48, 52, 55]. The possibility that human decision making is a rational outcome of neural processes has fostered the development of a new field, neuroeconomics [21]. However, human decision making is anything but rational. Clearly decision making also depends on past history [55], the emotional state [45] and the pressure that surrounds the decision making process [2, 3]. The fragile nature of human decision making reaffirms its dynamic basis [29].

Current mathematical models for decision making focus on decision making under ideal conditions. Thus it is assumed that each neural population is selectively responsive to components of the stimulus related to its decision and hence a winner can be chosen either based on neural firing rates ("forced response paradigm") or time to cross a threshold ("free response paradigm"). Implicit in this interpretation is the assumption that the inputs to the neural populations contain only information relevant for optimal decision making. However, recent fMRI $[37,38]$ and behavioral $[2,3]$ studies suggest that when decision making breaks down, e.g. "choking", the inputs to both neural populations contain a high proportion of irrelevant information. If, as a result, the inputs to each populations become sufficiently large then it is possible that both decisions can co-exist, i.e. the decision making neural network becomes bistable. Indeed, recent studies indicate that the dependence of performance and 
response time for monkeys on the degree of task difficulty in a perceptual decision making task are consistent with dynamics in the vicinity of the unstable saddle-type steady state which lies in the separatrix, i.e. the boundary between two attractors $[54,56]$.

An illustrative example is the changing perception of the Necker cube shown schematically in Figure 1. Here, each of the two perceptions represents one of the two wells in a double well potential and the ridge that separates the two wells is the separatrix $[4,6,13,29,43]$. In bistable dynamical systems which do not contain time delays, switches between the two wells require the action of external perturbations, typically in the form of "noise". However, time delays are present in all spatially distributed neural networks since axonal conduction velocities are finite. Consequently, decision making depends not only on the state of the neural population at the time the stimulus was provided but also on the activity of the network before the stimulus was presented [41, 42]. Thus it becomes possible in a timedelayed bistable system for trajectories to cross the boundary separating two attractors even in the absence of external perturbations such as noise. We hypothesize that at least some aspects of the fragility of human decision making are related to the effects of the separatrix in a time-delayed, bistable neural network.

Here we review the decision-making properties of a noisy time-delay differential equation model of a decision-making neural network composed of two mutually inhibiting neurons. Since this two-neuron network displays many of the same dynamical behaviors observed in larger networks [42], it provides an excellent starting point to understand the effect of delays and noise on decision making. For sufficiently high inputs there are three equilibria (two stable, one unstable). Although the stability of these equilibria is independent of the delay, the past history of the network nonetheless has an influence on current decision making [42]. When the function which describes the previous history of the state, i.e. the "initial function", is chosen to force the dynamics across the separatrix, the network becomes indecisive, i.e. the trajectories become transiently confined to the neighborhood of the separatrix. In this situation the effect of noise is to cause the network to make decisions by chance. Since time delays and noise are ubiquitous in the nervous system our observations suggest that these phenomena may lie at the basis of the observed fragility of human decision making.

\section{Fragility of human decision making}

What are the mechanisms that lead to suboptimal decision making? Neuro-cognitive science offers two theories for suboptimal decision making (for a review see Reference [3]). Each of these theories is based on the empirical suggestion that there is an "upside-down U" dependence of performance on attention [17]. In other words, optimal performance requires optimal attention. Self focus or explicit monitoring theories posit that performance becomes suboptimal because the pressure of the situation causes too much attention to be given to performance processes and procedures. In contrast, distraction theories posit that pressure fills working memory with distracting information so that attention to those details of the situation essential for optimal performance decreases. 
The difficulty with these interpretations is the lack of mechanistic explanations for the relation between performance and attention. Here we draw analogies between decision making in motor and cognitive tasks in order to obtain a possible mechanistic explanation for the relationships between performance and attention. Our interpretations are motivated by recent studies related to the performance of complex motor tasks, such as stick balancing at the fingertip and the maintenance of balance during quiet standing [9, 36, 39]. Decisions made for motor $[26,38,39]$ and cognitive [47, 52] tasks both emerge from distributed neural networks. Although the details of the involved networks differ, we anticipate that the underlying organizational principles for decision making share similar features. An advantage offered by the investigations of motor performance is that precise measurements of movements can be made using modern motion capture technologies. This greatly facilitates comparisons between observations and predictions of models based on Newton's laws of classical mechanics.

At issue is the distinction between skill and expertise [16,37]. Skill refers to the variability measured when the same motor task is repeated over and over by an individual, whereas the term expertise refers to the ability to maintain a given skill level over a range of situations. The decision making aspect of motor control arises because movements are controlled both by passive mechanisms related to biomechanical properties of the musculoskeletal system and the environment as well as actively by intentional corrective movements programmed by the nervous system (for reviews see References [35, 37]). How is the relative proportion of passive and active control decided upon? Several lines of investigation suggest that the development of expertise involves two steps [37, 39]: 1). an increasing reliance on the passive controlling mechanisms, and 2) a minimization of the role of active control mechanisms. The interplay between these two processes implies that, at a given skill level, performance decreases whenever active control becomes dominant. In the words of athletic coaches, performance decreases as the focus of the player shifts from goal to process $[16,17]$.

An often overlooked, but important reason for the trade-off between performance and the relative balance between passive and active control stems from the fact that neural control mechanisms for the regulation of motor tasks are time-delayed. In particular, there are fundamental limitations for control in the presence of time delays and noise [36, 50]. The problem can be readily appreciated by considering how those fluctuations in the controlled variable that must be acted upon by the controller can be distinguished from those which do not. This is because, by definition, there is a finite probability that any displacement from the set point will be counter-acted by a displacement towards the set point just by chance. Since there is a time delay, too quick a response by the controller to a given deviation can lead to the phenomenon of over-control leading to destabilization, particularly when the time delays are appreciable. On the other hand, waiting too long runs the risk that corrective movements are applied too late to be effective. Consequently when active control mechanisms are employed they will be ineffective and hence performance decreases. One way to overcome these problems is to use a switch-like, or discontinuous, feedback controller which is activated only when dynamical variables cross pre-set thresholds [36]. In other words, for small deviations of the control variable away from the set point, the control is 
passive, with active control exerted only once a threshold is crossed.

Insight into the nature of those aspects of attention that affect motor performance have been obtained from studies that compare novice and elite athletes [25, 38]. We illustrate these issues through a recent fMRI study that compared neural activity of novice and elite golfers during the preparatory period ("pre-shot routine") that precedes their golf shot [37, 38]. Overall central motor programs are more efficiently organized for elite golfers than novices, i.e. they require less energy [24]. However, this does not tell the whole story. The networks of novice and elite golfers are structurally different indicating that the novice brain seems to be solving a different, and likely more complex, problem than the expert [39]. Indeed behavioral observations of novice golfers during their pre-shot routine suggest that they are unnecessarily preoccupied with details that were irrelevant for this task [38]. Consistent with this interpretation was the observation that novice golfers activated brain regions associated with this type of filtering task, such as the posterior cingulate. Thus the poor performance of novice golfers may arise because too much attention is being placed on irrelevant details and hence they are trying to program a much more difficult task. This type of stress, i.e. an increase in the relative amount of irrelevant information, is very different from definitions of stress that emphasize the degree of activation of the autonomic nervous system [40].

\section{Decision making neural network}

In this paper, we consider a decision making neural network consisting of two mutually inhibitory neurons as depicted in Figure 2. A time-delayed differential equation model for this network is given by

$$
\begin{aligned}
& T_{1} \dot{x}=-x-S_{2}\left(y\left(t-\tau_{2}\right)\right)+I_{1}(t) \\
& T_{2} \dot{y}=-y-S_{1}\left(x\left(t-\tau_{1}\right)\right)+I_{2}(t)
\end{aligned}
$$

where $x, y$ are the firing rates of the neurons, $T_{1}, T_{2}$ are the neural time constants, $I_{1}, I_{2}$ represent external inputs, $\tau_{1}, \tau_{2}$ are the conduction time delays between the two neurons, and

$$
S_{j}(u)=\frac{c_{j} u^{n_{j}}}{\theta_{j}^{n_{j}}+u^{n_{j}}} \quad j=1,2
$$

describe sigmoidal functions representing inhibitory influences. Note that these functions are nonnegative on $u \geq 0$ and increasing. All constants in the model are positive except the delays which are nonnegative. The stability of this model has been discussed previously, particularly in the bistability regime with respect to the nature of the boundary, or separatrix, that separates the basins of attractors $[41,42]$. Here our interest concerns the effects of the initialization of this network on its behavior. For this purpose it is convenient to define both the initial conditions at $t=0$, i.e. $x(0)=X_{0}$ and $y(0)=Y_{0}$, and the initial functions $x(s)=X(s)$ and $y(s)=Y(s)$ for $s \in[-\tau, 0)$, where $\tau=\max \left(\tau_{1}, \tau_{2}\right)$. In this way the decision problem is represented by $\left(X_{0}, Y_{0}\right)$ and the past decision making history of the network is represented by $(X(s), Y(s)), s \in[-\tau, 0)$. 


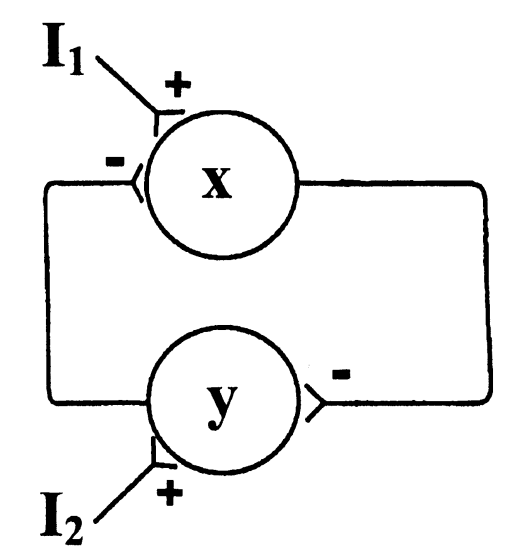

Figure 2: Schematic representation of a neural network with mutually inhibitory neurons. The '-' indicates an inhibitory connection and ' + ' indicates an excitatory connection. See text for discussion.

\subsection{Existence of Equilibrium Points}

We illustrate our hypothesis using constant inputs. In this case the system admits equilibrium points. An equilibrium point $\left(x^{*}, y^{*}\right)$ of $(3.1)$ must satisfy

$$
\begin{aligned}
& x^{*}=I_{1}-S_{2}\left(y^{*}\right)=f_{2}\left(y^{*}\right) \\
& y^{*}=I_{2}-S_{1}\left(x^{*}\right)=f_{1}\left(x^{*}\right) .
\end{aligned}
$$

Substituting (3.3) in (3.2) yields a single equation for $x^{*}$

$$
F(x) \stackrel{\text { def }}{=} I_{1}-S_{2}\left(I_{2}-S_{1}\left(x^{*}\right)\right)-x^{*}=0
$$

Now $\lim _{x \rightarrow \infty} F(x)<0$ since $S_{2}$ is nonnegative. Thus if $F(0)=I_{1}-S_{2}\left(I_{2}\right)>0$, then $F(x)$ will have at least one root with $0<x^{*}<I_{1}$. Consideration of (3.3) shows that the root will also have $y^{*}>0$ if $I_{2}-S_{2}\left(I_{1}\right)>0$. Thus, if these two conditions are satisfied there will be at least one equilibrium in the first quadrant. Note that the same argument also holds for any nonlinearities, $S_{j}$, which are nonnegative and increasing.

With the nonlinearity we have chosen, when the conditions defined above are satisfied, we observe that there can be one, two or three equilibria in the first quadrant. We focus on the situation when there are three equilibria in the first quadrant (Figure 3). This corresponds to assuming that the mean value of $I_{1}$ and $I_{2}$ is sufficiently high as a result of input containing a high proportion of information that is not required for optimal decision making, but nonetheless is detected by the neural population (Section 2). 


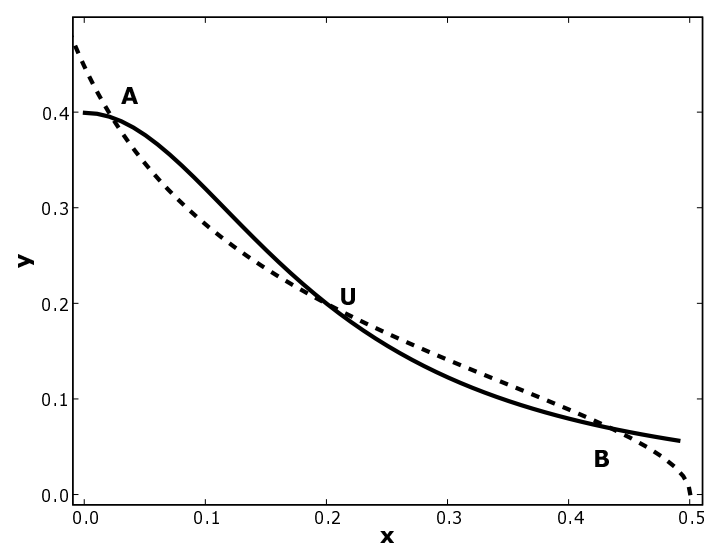

Figure 3: Determination of the equilibria for (3.1). The solid line corresponds to $y^{*}=f_{1}\left(x^{*}\right)$ and the dashed line to $x^{*}=f_{2}\left(y^{*}\right)$ (see (3.2)-(3.3)). There are three positive equilibria for this choice of parameters: the unstable equilibrium is labeled ' $U$ ', and the two stable equilibria are 'A' and 'B'. Parameter values: $T_{1}=T_{2}=1, c_{1}=0.4, c_{2}=0.6, I_{1}=0.5$, $I_{2}=0.4, n_{1}=2, n_{2}=2, \theta_{1}=0.2$, and $\theta_{2}=0.2$.

\subsection{Stability}

Consider an equilibrium point $\left(x^{*}, y^{*}\right)$ of (3.1). The linearization of (3.1) about this equation is given by

$$
\begin{aligned}
& T_{1} \dot{x}=-x-S_{2}^{\prime}\left(y^{*}\right) y\left(t-\tau_{2}\right) \\
& T_{2} \dot{y}=-y-S_{1}^{\prime}\left(x^{*}\right) x\left(t-\tau_{1}\right) .
\end{aligned}
$$

The stability of the equilibrium point may be found as follows. Consider the nontrivial solutions of (3.5), which are of the form $(x(t), y(t))=e^{-\lambda t}(v, w)$, where $v, w$ and $\lambda$ are constants. Substituting this form into (3.5) yields a linear system for $v$ and $w$. Requiring that that this system has nontrivial solutions, i.e., $v \neq 0, w \neq 0$, yields the characteristic equation

$$
T_{1} T_{2} \lambda^{2}+\left(T_{1}+T_{2}\right) \lambda+1-S_{1}^{\prime}\left(x^{*}\right) S_{2}^{\prime}\left(y^{*}\right) e^{-\lambda\left(\tau_{1}+\tau_{2}\right)}=0 .
$$

Any $\lambda$ which is a root of this equation will determine a solution of the form given above. If all such solutions satisfy $\operatorname{Re}(\lambda)<0$ then the equilibrium point is asymptotically stable. If at least one solution satisfies $\operatorname{Re}(\lambda)>0$ then the equilibrium point is unstable.

For simplicity, we define $\tau_{12}=\left(\tau_{1}+\tau_{2}\right) / 2$ and, since $S_{j}^{\prime}(u) \geq 0$ for all $u$, we define $\gamma^{2}=S_{1}^{\prime}\left(x^{*}\right) S_{2}^{\prime}\left(y^{*}\right)$. Then the characteristic equation becomes

$$
T_{1} T_{2} \lambda^{2}+\left(T_{1}+T_{2}\right) \lambda+1-\gamma^{2} e^{-2 \lambda \tau_{12}}=0 .
$$

When $\tau_{12}=0,(3.7)$ can be solved for the two roots

$$
\lambda_{ \pm}=\frac{-\left(T_{1}+T_{2}\right) \pm \sqrt{\left(T_{1}+T_{2}\right)^{2}-4\left(1-\gamma^{2}\right) T_{1} T_{2}}}{2 T_{1} T_{2}} .
$$


It is easy to see that both roots will have negative real parts if $\gamma^{2}<1$, while there will be one positive and one negative real root if $\gamma^{2}>1$. Thus we conclude that for zero delay, the equilibrium point will be asymptotically stable if

$$
S_{1}^{\prime}\left(x^{*}\right) S_{2}^{\prime}\left(y^{*}\right)<1
$$

and unstable if

$$
S_{1}^{\prime}\left(x^{*}\right) S_{2}^{\prime}\left(x_{2}^{*}\right)>1
$$

On the graphical representation of the equilibrium points given in Figure $3, S_{1}^{\prime}\left(x^{*}\right)$ is the magnitude of the slope of the solid curve at the intersection point and $1 / S_{2}^{\prime}\left(y^{*}\right)$ is the magnitude of the slope of the dashed curve at the intersection point. Thus simple graphical conditions for stability are

- $\left(x^{*}, y^{*}\right)$ is asymptotically stable if the magnitude of the slope of the solid curve is less than the magnitude of the slope of the dashed curve at $\left(x^{*}, y^{*}\right)$,

- $\left(x^{*}, y^{*}\right)$ is unstable if the magnitude of the slope of the solid curve is greater than the magnitude of the slope of the dashed curve at $\left(x^{*}, y^{*}\right)$.

From this, it is clear that in Figure 3 the middle equilibrium point is unstable, while the other two are asymptotically stable.

In fact the situation shown in Figure 3 is always the case when there are three equilibrium points in the first quadrant, as we now show. Recall the function $F(x)$ given by (3.4). At an equilibrium point $\left(x^{*}, y^{*}\right)$, we have

$$
\begin{aligned}
F^{\prime}\left(x^{*}\right) & =S_{2}^{\prime}\left(I_{2}-S_{1}\left(x^{*}\right)\right) S_{1}^{\prime}\left(x^{*}\right)-1 \\
& =S_{2}^{\prime}\left(y^{*}\right) S_{1}^{\prime}\left(x^{*}\right)-1 .
\end{aligned}
$$

Thus the equilibrium point will be asymptotically stable if $F^{\prime}\left(x^{*}\right)<0$ and unstable if $F^{\prime}\left(x^{*}\right)>0$. Now suppose that the conditions given in section 3.1 for the existence of an equilibrium point in the first quadrant are satisfied and there are three equilibrium points with $x$ coordinates $0<x_{1}^{*}<x_{2}^{*}<x_{3}^{*}$. A simple geometric argument shows that necessarily $F^{\prime}\left(x_{1}^{*}\right)<0, F^{\prime}\left(x_{2}^{*}\right)>0$ and $F^{\prime}\left(x_{3}^{*}\right)<0$, so the inner equilibrium point is unstable and the other two are stable.

In the situation where there are two co-existing asymptotically stable equilibrium points in the system, the question arises: Given an initial condition $\left(X_{0}, Y_{0}\right)$ for equation (3.1) with no delay $\left(\tau_{1}=\tau_{2}=0\right)$, which equilibrium point will the corresponding solution approach? For a two dimensional system such as (3.1) this question is easily answered. The stable manifold of the saddle-type equilibrium point ( $U$ in Figure 3$)$ is a separatrix, i.e. it separates the $x, y$ plane into regions. Initial conditions which lie to the left of the the separatrix yield solutions which asymptotically approach equilibrium point $A$ and those which lie to the right approach $B$. These regions are called the basins of attraction of the equilibrium points $A$ and $B$. 
If $\tau_{12}>0$ there is a countable infinity of roots of the characteristic equation (3.7), thus one must use other means to study the stability of equilibrium points. In particular, we will use the following result [28, 49]: As $\tau_{12}$ is increased from zero, the number of roots of (3.7) with positive real parts can only change if some roots pass through the imaginary axis.

Consider first the case $\gamma^{2}<1$. When $\tau_{12}=0$ both roots of (3.7) have negative real parts and the equilibrium point, $\left(x^{*}, y^{*}\right)$ is asymptotically stable. Using the result above, we conclude that there can only be a change of stability of the equilibrium point at a value of $\tau_{12}$ such that $\lambda=i \omega$ is a root of (3.7). Substituting this into (3.7) and separating the result into real and imaginary parts yields the following equations

$$
\begin{aligned}
1-T_{1} T_{2} \omega^{2} & =\gamma^{2} \cos 2 \omega \tau_{12}, \\
\left(T_{1}+T_{2}\right) \omega & =-\gamma^{2} \sin 2 \omega \tau_{12} .
\end{aligned}
$$

Squaring and adding these equations yields the following condition on $\omega$

$$
1+\left(T_{1}^{2}+T_{2}^{2}\right) \omega^{2}+T_{1}^{2} T_{2}^{2} \omega^{4}=\gamma^{4} .
$$

Since $\gamma^{2}<1$. This implies that this condition cannot be satisfied for any $\omega$. We conclude that there are no pure imaginary roots of (3.7) for any value of $\tau_{12}>0$ and the equilibrium point must remain asymptotically stable for all $\tau_{12}>0$.

Now consider the case $\gamma^{2}>1$. for $\tau_{12}=0$, one root of (3.7) is positive and thus $\left(x^{*}, y^{*}\right)$ is unstable. Consider just the real roots, $\lambda$, of (3.7) when $\tau_{12}>0$. These must satisfy $g(\lambda)=0$ where $g(\lambda)=T_{1} T_{2} \lambda^{2}+\left(T_{1}+T_{2}\right) \lambda+1-\gamma^{2} e^{-2 \lambda \tau_{12}}$. Now $g(0)=1-\gamma^{2}<0$ and $\lim _{\lambda \rightarrow \infty} g(\lambda)>0$. Hence, since $g(\lambda)$ is continuous, there must be a $\lambda>0$ such that $g(\lambda)=0$ for all $\tau_{12}>0$. Thus $\left(x^{*}, y^{*}\right)$ is unstable.

Finally, we note that when $\gamma^{2}=1$ the characteristic equation (3.7) has a zero root for $\tau_{12} \geq 0$, thus the stability is not determined by the linearization.

In summary, for $\gamma^{2} \neq 1$, the stability of equilibrium points is unaffected by the presence of the delay and can determined for any $\tau_{1} \geq 0, \tau_{2} \geq 0$ by the slopes of the nonlinearities at the equilibrium point.

It should be noted that when there are three equilibria in the first quadrant it is possible to write (3.1) in the form of two mutually excitatory neural populations [41, 42]. In this case it was shown by Pakdaman et al. [42], using the theory of monotone dynamical systems, that the stability of the three equilibria is independent of the delay.

While the stability of the equilibrium points is unaffected by the presence of the delay, describing the basins of attraction of the equilibrium points becomes considerably more complex. To see this, note that when the delays are zero, the basins are subsets of the plane. However, when at least one delay is not zero, the basins are subsets of an infinite dimensional space consisting of the set of all initial functions for the delay differential equation. Pakdaman et al. [42] have studied the basins of attraction for (3.1) by considering a restricted set of initial functions. Even with this restriction they found that the delay could have profound effects on the basins of attraction. In the following we will explore the implications of this when (3.1) is interpreted as a model for decision making. 


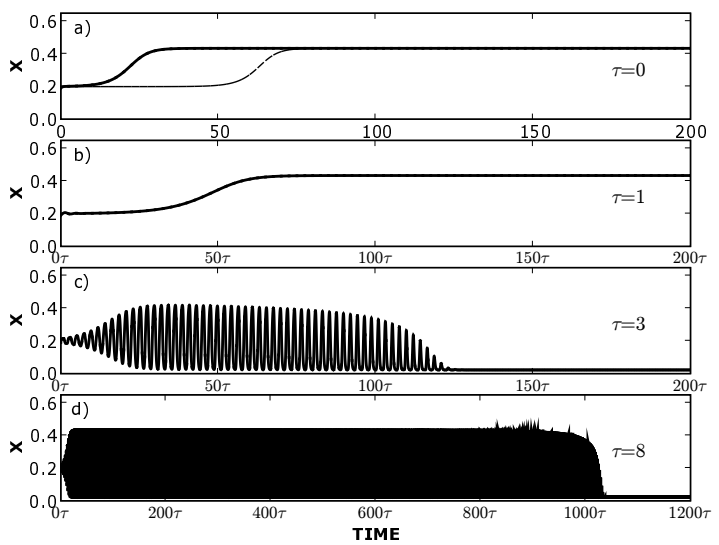

Figure 4: Dynamics of (4.1) as a function of time delay under conditions when three equilibria co-exist. When $\tau=0$ the initial condition was either $(0.19,0.19)$ (solid line) or $(0.199999,0.199999)$ (dotted line). When $\tau \neq 0$ the initial condition was $(0.19,0.19)$ and the initial function was $(X(s), Y(s))=(0.19,0.19)$ for $s \in[-\tau, 0)$. Values of the parameters are the same as in Figure 3. In order to facilitate comparison to the no delay case in a) in b), c), and d) we multiplied the time unit by $\tau$.

\section{Decision making}

In the remainder of the paper we focus on the case where three equilibria co-exist. For simplicity we take $\tau \equiv \tau_{1}=\tau_{2}$. Using the change of independent variable $t \rightarrow t / \tau$, (3.1) can be re--written as

$$
\begin{aligned}
& \dot{x}=-\tau^{\prime} x-\tau^{\prime} S_{2}(y(t-1))+\tau^{\prime} I_{1} \\
& \dot{y}=-\tau^{\prime} y-\tau^{\prime} S_{1}(x(t-1))+\tau^{\prime} I_{2}
\end{aligned}
$$

where we have taken $T \equiv T_{1}=T_{2}$ and $\tau^{\prime}=\tau / T$.

\subsection{Past history and decision making}

When $\tau=0$ the decision made by the network corresponds to the choice of the initial condition $\left(X_{0}, Y_{0}\right)$. Thus if $\left(X_{0}, Y_{0}\right)$ lies to the left of ' $\mathrm{U}$ ' in Figure 3 the decision will be A, and if $\left(X_{0}, Y_{0}\right)$ lies to the right the decision will be B. We choose $(0.19,0.19)$ to be close, but left of ' $U$ ', and ask how long does it takes the solution to settle onto decision A. Figure 4a shows that it takes 25-35 time units for the solution corresponding to this initial condition to settle on decision A. It should be noted that since the separatrix is the stable manifold of a saddle-type equilibrium point, the closer we choose $\left(X_{0}, Y_{0}\right)$ to the separatrix the longer it takes the network to settle on a decision (see dotted line in Figure 4a).

When $\tau \neq 0$ the decision by the network is determined by the initial condition $\left(X_{0}, Y_{0}\right)$ and the initial function $(X(s), Y(s)), s \in[-\tau, 0)$. There are two consequences. The first 
consequence is that the behavior close to the separatrix is changed. This can be illustrated by choosing $\left(X_{0}, Y_{0}\right)=(0.19,0.19)$ as in Figure 4a and the constant initial function $(X(s), Y(s))=(0.19,0.19), s \in[-\tau, 0)$ and then varying $\tau$. When $\tau=1$ the time it takes the solution to settle monotonically onto decision A is slightly longer, about $55-60 \tau$ time units (compare Figure 4a to Figures 4b,c,d). However when $\tau$ is larger the transient state becomes oscillatory and it takes longer to settle on a decision; in this case decision B (compare Figure $4 \mathrm{~b}$ to Figure 4c,d). The length of the oscillatory transients, or metastable states $[23,41]$, increases (compare Figure 4c to Figure 4d). These oscillatory metastable states correspond to a type of indecisiveness in which the network wavers between two decisions before finally choosing one. A surprising observation that the time spent wavering can be much longer than the delay $\tau$.
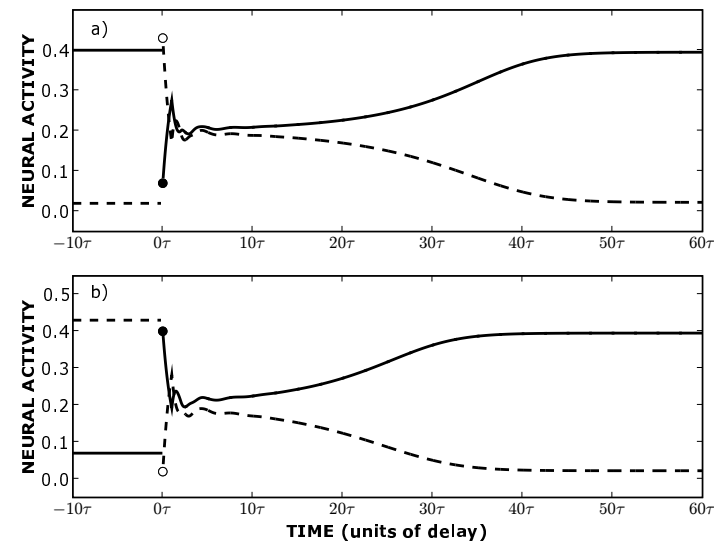

Figure 5: Influence of past history on the decision making behavior of (3.1). As discussed in the text, the initialization has been divided for convenience into an initial condition at $t=0$ and an initial function for $t \in[-\tau, 0)$. a) The initial function is chosen to be constant near the 'A' decision, $(0.02,0.4)$, (see Figure 3 ) and the initial condition is taken to be near the ' $\mathrm{B}$ ' decision, $(0.43,0.07)$. b) The initial function is chosen to be constant near the ' $\mathrm{B}$ ' decision, $(0.43,0.07)$, and the initial condition is taken to be near the 'A' decision, $(0.02,0.4)$. The solid line is $y(t)$ and the dashed line is $x(t)$.

The second consequence is that the past history affects the decision making process described by (4.1). This can be illustrated by choosing $(X(s), Y(s))$ to place the network in one basin of attraction and then determining the effect of different choices of $\left(X_{0}, Y_{0}\right)$ in the other basin of attraction. The important point is that by choosing $\left(X_{0}, Y_{0}\right)$ and $(X(s), Y(s))$ to correspond to different sides of the separatrix, the solution trajectories are forced to cross the separatrix. In Figure 5a the initial function is close to $\mathrm{A}$ and the initial condition is close to $\mathrm{B}$. In this situation, the network still reaches the decision A, unlike what would occur with $\tau=0$. We refer to such a situation as an incorrect decision. In Figure 5b the initial function is chosen to be close to decision $\mathrm{B}$ and the initial condition to be close to decision A. In this case the network obtains the same solution as would be obtained when $\tau=0$, 


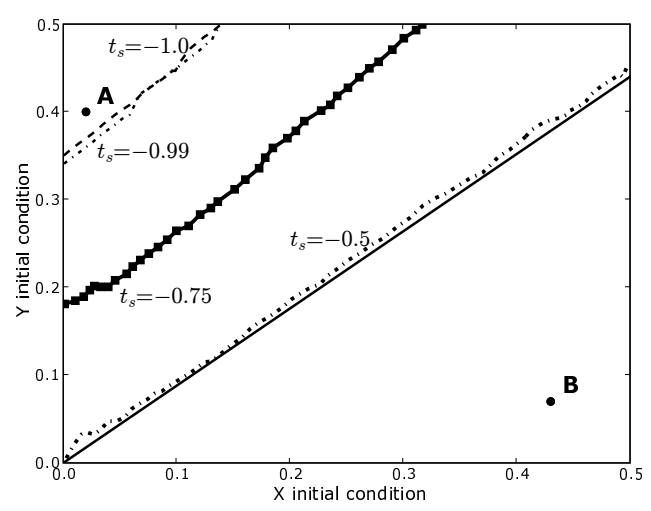

Figure 6: Relationship between the initial function, the initial condition, and decision making by (4.1). The initial functions were constructed using (4.2). For each choice of the initial function constructed by choosing $t_{s}$, the initial values of $x$ and $y$ were varied in order to locate the position of the separatrix. The area to the right of each separatrix corresponds to initial conditions that resulted in decision $\mathrm{B}$, i.e. as $t_{s}$ increases the initial choices of $x$ and $y$ that result in B increases. The solid line shows the separatrix when $\tau=0$.

namely A. It should be noted that in each case the solution initially becomes confined near the boundary than separates the two decisions, i.e. $(0.19,0.19)$, and thus it takes $30-35 \tau$ for the network to settle on its decision.

Figure 6 summarizes the decision making behavior of (4.1) as a function of constant past histories of the type used in Figure 5. This figure was constructed by the choosing initial function

$$
(X(s), Y(s))=\left\{\begin{array}{l}
A \text { if }-1 \leq s<t_{s} \\
B \text { if } t_{s} \leq s<0
\end{array}\right.
$$

where $t_{s}$ is a time that divides the initial function into two parts, and then running simulations varying the initial condition $\left(X_{0}, Y_{0}\right)$. For each value of $t_{s}$ a separatrix is shown, which divides the initial conditions into those which lead to decision A (left of the separatrix) from those which lead to decision $\mathrm{B}$ (right of the separatrix). When $t_{s}=-0.5$ the location of the separatrix is approximately the same to that obtained when $\tau=0$ (dotted line). As $t_{s} \rightarrow-1$, the separatrix shifts towards the $A$ decision. For any $t_{s}<-0.5$, incorrect decisions (i.e. B) are made when $\left(X_{0}, Y_{0}\right)$ are chosen within the area between the separatrix for the current $t_{s}$ and that for $t_{s}=-0.5$.

\subsection{Choking}

Our hypothesis is that choking occurs when inputs to both neural populations involved in the decision making process are high and the inputs contain both relevant and irrelevant information (see Section 2). It is reasonable to model the irrelevant information as additive 
random perturbations, i.e. "noise", and thus (4.1) becomes

$$
\begin{aligned}
& \dot{x}=-\tau^{\prime} x-\tau^{\prime} S_{2}(y(t-1))+\tau^{\prime} I_{1}+\sigma \xi(t) \\
& \dot{y}=-\tau^{\prime} y-\tau^{\prime} S_{1}(x(t-1))+\tau^{\prime} I_{2}+\sigma \xi(t)
\end{aligned}
$$

where $\xi(t)$ describes uniformly distributed "white" noise.

The dynamics exhibited by bistable, retarded dynamical systems acting under the influence of noise are very complex (see, for example, $[9,15,19,23]$ ), and are not yet completely understood. Here we draw attention to the effects of noise for different choices of the initial function and condition (Figure 7). Overall noise does not eliminate the indecisive state, but can affect the decision outcome. Figure 7a shows consecutive realizations of (4.3) when the initial condition and function are chosen for the case when the decision making network makes the incorrect decision (Figure 5a). Although high intensity noise $(\sigma=0.3)$ can cause the network to make the correct decision (3 out of 14 consecutive trials) it remains biased towards making the incorrect decision. A bias towards the solution determined by the choice of initial function is also seen when the network continues to make the correct decision even in the presence of high noise $(\sigma=0.3$; Figure $7 \mathrm{~b})$. However, a very different result is obtained when the initial function and condition are chosen to place the trajectories near the boundary separating the two basins of attraction for at least time $\tau$, i.e. during the period of time when the network is indecisive (Figure 7c). In this case the effect of the irrelevant information during this critical period is to cause the network to randomly reach its decisions: sometimes it chooses A, other times B. Overall the number of times the network chooses $\mathrm{A}$ is about the same number of times it chooses B, i.e. the decision making ability of the network under these conditions is no better than chance.

\section{Discussion and conclusions}

Our observations suggest a scenario for the failure of a decision making neural network. A condition for failure is that the stimulus for both neural populations $\left(I_{1}\right.$ and $\left.I_{2}\right)$ must be greater than the magnitude of the inhibitory interactions between them (given, respectively, by $c_{2}$ and $c_{1}$ ). Consequently the decision making neural network exhibits bistability, i.e. both decisions (attractors) co-exist. In the context of this hypothesis, the changing interpretation of ambiguous figures, such as the Necker cube in Figure 1, would arise because the input to both neural populations is simultaneously high enough. A mathematical requirement for the existence and uniqueness of solutions in a bistable dynamical system is that every pair of attractors must be separated by an unstable object. Typically this takes the form of a saddle-type object, with the stable manifold of this object forming a separatrix between the basins of attraction of the two attractors. Since the decision making neural network is time-delayed, it is possible that the initial condition and the initial function can lie, at least in part, on opposite sides of the separatrix. As a result the decision making behaviors of the network become dominated not only by the presence of the attractors, but also by the presence of the saddle object. In particular the trajectories can become transiently confined 

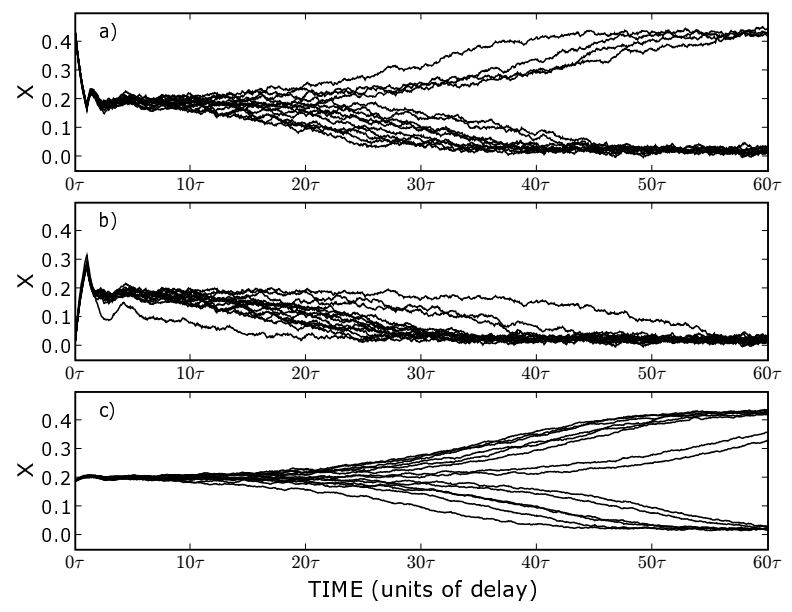

Figure 7: Effect of irrelevant information on decision making for different choices of the initial function and initial condition: a) the initial function and condition as in Figure 5a; b) the initial function and condition as in Figure 5b; and c) the indecisive regime, i.e. the initial function and condition are both (0.19.0.19), i.e. close to ' $U$ ' in Figure 3. The irrelevant information consists of additive white noise, uniformly distributed on $[-0.5,0.5]$ with $\sigma=0.3$ in a) and b) and $\sigma=0.1$ in c). Remaining parameters are the same as used in Figure 5.

for times longer than $\tau$ in the near neighborhood of the separatrix (see also Reference [23]), i.e. the network becomes temporarily indecisive. When the noise level is very low, the effect is to cause history-dependent errors in current decision making. When the noise level is sufficiently high and depending on the choice of initial function and condition, it becomes possible that decisions are made by chance. This is in contrast to bistable systems with no delay $(\tau=0)$ : in this case even low noise can blur the boundary between basins of attraction of the stable states thus effectively eliminating the influence of the initial condition. Our results suggest that for bistable dynamical systems with delay the influence of past history is not necessarily destroyed by noise.

Central neural conduction time delays are typically of the order of 10-100 milliseconds, see, for example, [22, 33]. However, psycho-physical and neurophysiological measurements suggest that decision making takes much longer, for example hundreds of msec [5, 54, 56]. One explanation for the long time to make decisions is that it reflects slow dynamics of certain receptors, such as the NMDA receptor [56]. However, our modeling efforts emphasize that the slowness of the decision making process may be related to the properties of a timedelayed and bistable dynamical system. A consequence of time delays in a bistable system is the generation of long-lived metastable states [41] and hence the time it takes to make a decision can be very much larger than the time delay. Thus decision making provides an example of a neural network behavior that is largely shaped by the effects of the unstable steady states. Other examples in which dynamics are shaped by similar instabilities occur 
in stick balancing at the fingertip [9] and reverse accommodation of neurons [1, 44]. These effects become particularly important when there is an interplay between time delays and noise [36].

Decisions made by the nervous system are thought to be made on the basis of both internally-guided and externally-guided influences $[11,46]$. A vexing problem has been to understand why decision errors arise when these two influences are in conflict. This is particularly true in human subjects with frontal lobe dysfunction who perseverate, i.e. subjects who continue to repeat a previously appropriate response even though the repeated response is no longer appropriate $[12,34]$. Qualitatively similar, but typically much less severe, responses arise in many decision making contexts ranging from the interaction between visual and anticipatory biases in adaptive decision making tasks in primates [11] to the voting trends in presidential elections [55]. Previous explanations have either attributed these phenomena to the role played by different anatomical loci $[11,34]$ or, in the context of neural networks, to the weakening of signals between sensory and reinforcement loci [30]. Here we have used a simple decision making neural network with delay to argue that past history can effect current decision making behaviors, particularly when multiple decisions co-exist. In the context of a delay differential equation model such as ours, the past history is represented by the initial function. Unfortunately the effects of the choice of initial function on the behavior of delay differential equations have received surprisingly little attention. Notable exceptions are the work of Foss et al. [18, 19], Losson et al. [31], Milton et al. [36], Pakdaman et al. [41, 42], and Takác [51]. Thus an important first step in translating from this simple model to experimental investigations at the benchtop will be the development of mathematical tools to explore the role of the initial function in shaping the behavior of a retarded dynamical system. One difficulty is that software packages for integrating delay differential equations are typically capable of handling only a constant-valued initial function. An exception is the software package, XPPAUT [14]; however, for this program the manner in which an arbitrary initial function can be incorporated is not well documented. In the Appendix we show how this is done. The goal in developing such tools will be to identify strategies that exploit the dependence on the initial function of the dynamics of time-delayed mathematical models in order to devise experiments to determine whether effects similar to those described here can be demonstrated experimentally.

The effects of past history and noise arise in any real spatially distributed dynamical system in which time delays are a necessary component. Moreover, behaviors, even more complex than we have discussed here, can arise in situations in which decisions are not represented by equilibria, but rather correspond to other types of bounded, time-dependent states such as a limit cycle (see, for example, References [15, 20, 23]). In any case we suggest that studies of decision making under conditions where this process become fragile are likely to be important for determining the underlying mechanisms for human decision making. 


\section{Acknowledgements}

The authors are grateful for useful discussions with T. Ohira and for XPPAUT programming assistance provided by B. Ermentrout. We acknowledge support from the National Science Foundation (NSF-0617072 (JM) and NSF-0634592 (PN,CC)) and the Natural Sciences and Engineering Research Council of Canada (SAC).

\section{References}

[1] S. M. Baer, T. Erneux, J. Rinzel. The slow passage through a Hopf bifurcation: Delay, memory effects and resonance. SIAM J. Appl. Math., 49 (1989), 55-71.

[2] S. L. Beilock, T. H. Carr. On the fragility of skilled performance: What governs choking under pressure? J. Exper. Psych.: Gen., 130 (2001), 701-725.

[3] S. L. Beilock, C. A. Culp, L. E. Holt, T. H. Carr. More on the fragility of performance: Choking under pressure in mathematical problem solving. J. Exp. Psych., 133 (2004), No. 4, 584-600.

[4] W. Bialek, M. De Weese. Random switching and optimal processing in the perception of ambiguous signals. Phys. Rev. Lett., 74 (1995), 3077-3079.

[5] R. Bogacz, E. Brown, J. Moehlis, P. Holmes, J. D. Cohen. The physics of optimal decision making: A formal analysis of models of performance in two-alternative forcedchoice tasks. Psych. Rev. (2006), 700-765.

[6] A. Borsellino, A. DeMarco, A. Allazetta, S. Rinsei, B. Bartolini. Reversal time distribution in the perception of visual ambiguous stimuli. Kybernetik, 10 (1972), 139-144.

[7] K. L. Briggman, H. D. I. Abarbanel, W. B. Kristan Jr. Optical imaging of neuronal populations during decision-making. Science 307 (2005), 896-901.

[8] E. Brown, J. Gao, P. Holmes, R. Bogacz, M. Gilzenrat, J. D. Cohen. Simple neural networks that optimize decisions. Int. J. Bifurc. Chaos, 15 (2005), No. 3, 803-826.

[9] J. L. Cabrera, J. G. Milton. On-off intermittency in a human balancing task. Phys. Rev. Lett., 89 (2002), 158702.

[10] P. J. Choi, L. Cai, K. Fieda, X. S. Xie. A stochastic single-molecule event triggers phenotype switching of a bacterial cell. Science, 322 (2008), No. 5900, 442-446.

[11] B. Coe, K. Tomihara, M. Matsuzawa, O. Hikosaka. Visual and anticipatory bias in three cortical eye fields of the monkey during an adaptive decision-making task. J. Neurosci., 22 (2002), 5081-5090. 
[12] K. J. Cole, D. L. Rotella. Old age impairs the use of arbitrary visual cues for predicitive control of fingertip forces during grip. Exp. Brain Res. 143 (2002), 35-41.

[13] G. Deco, M. Pérez-Sanagustin, V. de Lafuente, R. Romo. Perceptual detection as a dynamical bistability phenomenon: A neurocomputational correlate of sensation. Proc. Natl. Acad. Sci. USA, 104 (2007), 20073-20077.

[14] B. Ermentrout. Simulating, Analyzing, and Animating Dynamical Systems: A guide to XPPAUT for researchers and students. SIAM, Philadelphia, 2002.

[15] C. W. Eurich, J. G. Milton. Noise-induced transitions in human postural sway. Phys. Rev. E, 54 (1996), 6681-6684.

[16] M. Fairweather. Skill learning principles: implications for coaching practice. In: N. Cross, J. Lyle, eds, The Coaching Process: Principles and Practice for Sport. Butterworth Heinemann, New York, 1999, pp. 113-129.

[17] P. M. Fitts, M. I. Posner. Human performance. Brooks/Cole, Belmont, CA, 1967.

[18] J. Foss, A. Longtin, B. Mensour, J. G. Milton. Multistability and delayed recurrent loops. Phys. Rev. Lett., 76 (1996), 708-711.

[19] J. Foss, F. Moss, J. Milton. Noise, multistability, and delayed recurrent loops. Phys. Rev. E, 55 (1997), 4536-4543.

[20] W. J. Freeman, W. S. Schneider. Changes in spatial patterns of rabbit olfactory EEG with conditioning to odors. Psychophysiology, 19 (1982), 44-56.

[21] P. W. Glimcher, C. F. Camerer, E. Fehr, R. A. Poldrack, eds. Neuroeconomics: Decision-making and the Brain. Academic Press, New York, 2009.

[22] J. Gotman. Measurement of small time differences between EEG channels: method and application to epileptic seizure propagation. Electroencephalogr. Clin. Neurophysiol., 79 (1983), 403-412.

[23] C. Grotta-Ragazzo, K. Pakdaman, C. P. Malta. Metastability for delayed differential equations. Phys. Rev. E., 60 (1999), 6230-6233.

[24] B. D. Hatfield, A. J. Haufler, T.-M. Hung, T. W. Spalding. Electroencephalographic studies of skilled psychomotor performance. J. Clin. Neurophysiol., 21 (2004), 144-156.

[25] B. D. Hatfield, C. H. Hillman. The psychophysiology of sport: a mechanistic understanding of the psychology of superior performance. In: Handbook of Sport Psychology (R. N. Singer, H. A. Hausenblas, C. M. Janelle, eds). Wiley \& Sons, New York, 2001, pp. 362-386. 
[26] M. Jeannerod, J. Decety. Mental motor imagery: a window into the representational stages of action. Curr. Opin. Neurobiol., 5 (1995), 727-732.

[27] J. N. Kim, M. N. Shadlen, Neural correlates of a decision in the dorsolateral prefrontal cortex of the macaque. Nature Neuroscience, 2 (1999), 176-185.

[28] V. B. Kolmanovskii, V. R. Nosov, V. R. Stability of Functional Differential Equations. Academic Press, London, 1986.

[29] P. Kruse, M. Stadler, eds. Ambiguity in Mind and Nature: Multistable cognitive phenomena. Springer, New York, 1995.

[30] D. S. Levine, P. S. Prueitt. Modeling some effects of frontal lobe damage - novelty and preservation. Neural Net. 2 (1989), 103-116.

[31] J. Losson, M. C. Mackey, and A. Longtin, Solution multistability in first order nonlinear delay differential equations. Chaos 3 (1993), 167-176.

[32] M. E. Mazurek, J. D. Roitman, J. Ditterich, M. N. Shadlen. A role for neural integrators in perceptual decision making. Cereb. Cortex, 13 (2003), 1257-1269.

[33] R. Miller. What is the contribution of axonal conduction delay to temporal structure in brain dynamics?. In: Oscillatory Event-related Brain Dynamics (C. Pantev, ed). Plenum Press: New York, 1994, pp. 53-57.

[34] B. Milner. Some effects of frontal lobectomy in man. In: The frontal granular cortex and behavior (J. Warren, K. Akert, eds). McGraw-Hill: New York, 1964, pp. 313-334.

[35] J. Milton, ed. Focus Issue on Bipedal Locomotion: From robots to humans. Chaos, 19 (2009).

[36] J. G. Milton, J. L. Cabrera, T. Ohira. Unstable dynamical systems: Delays, noise and control. EPL, 83 (2008), 48001.

[37] J. G. Milton, S. S. Small, A. Solodkin. On the road to automatic: Dynamic aspects in the development of expertise. J. Clin. Neurophysiol., 21 (2004), no. 3, 134-143.

[38] J. Milton, A. Solodkin, P. Hlustik, S. L. Small. The mind of expert motor performance is cool and focused. NeuroImage, 35 (2007), 804-813.

[39] J. Milton, S. L. Small, A. Solodkin. Imaging motor imagery: Methodological issues related to expertise. Methods, 45 (2008), 336-341.

[40] K. Oishi, T. Maeshima. Autonomic nervous system activities during motor imagery in elite athletes. J. Clin. Neurophysiol., 21 (2004), 170-179.

[41] K. Pakdaman, C. Grotta-Ragazzo, C. P. Malta. Transient regime duration in continuous-time neural networks with delay. Phys. Rev. E, 58 (1998), 3623-3627. 
[42] K. Pakdaman, C. Grotta-Ragazzo, C. P. Malta, O. Arino, J. -F. Vibert. Effect of delay on the boundary of the basin of attraction in a system of two neurons. Neural Networks, 11 (1998), 509-519.

[43] M. Riani, E. Simonotto. Stochastic resonance in the perceptual interpretation of ambiguous figures: A neural network approach. Phys. Rev. Lett., 72 (1994), 3120-3123.

[44] J. Rinzel, S. M. Baer. Threshold for repetitive activity for a slow stimulus ramp: A memory effect and its dependence on fluctuations. Biophys. J., 54 (1988), 551-555.

[45] A. G. Sanfey, J. K. Rilling, J. A. Aronson, L. E. Nystrom, J. D.Cohen. The neural basis of economic decision-making in the ultimatum game. Science, 300 (2003), 1755-1758.

[46] J. D. Schall. Neural basis of deciding, choosing, and acting. Nat. Neurosci. 2 (2001), $33-42$.

[47] B. Seymour, N. Daw, P. Dayan, T. Somger, R. Dolan. Differential encoding of losses and gains in the human straitum. J. Neurosci., 27 (2007), 4826-4831.

[48] M. N. Shadlen, W. T. Newsome. Neural basis of a perceptual decision in the parietal cortex (area LIP) of the rhesus monkey. J. Neurophysiol., 86 (2001), 1916-1936.

[49] G. Stépán. Retarded Dynamical Systems: Stability and Characteristic Functions. Longman Group, Essex, 1989.

[50] G. Stépán, T. Insperger. Stability of time-periodic and delayed systems - a route to act-and-wait control. Annu. Rev. Control, 30 (2006), 159-168.

[51] P. Takác. Domains of attraction of generic omega-limit sets for strongly monotone semi-flows. Zeitschrift fur Analysis und ihre Answendungen, 10 (1991), 275-317.

[52] A. Thielscher, L. Pessoa. Neural correlates of perceptual choice and decision making during fear-disgust discrimination. J. Neurosci., 27 (2007), 2908-2917.

[53] M. Usher, J. L.McClelland. The time course of perceptual choice: The leaky, competing accumulator model. Psychol. Rev., 108 (2001), 550-592.

[54] X.--J. Wang. Probabilistic decisions making by slow reverberation in cortical circuits. Neuron, 36 (2002), 955-968.

[55] D. Westen, P. S. Blagov, K. Harenski, C. Kilts, S. Hamann. Neural bases of motivated reasoning: An fMRI study of emotional constraints on partisan political judgement in the 2004 U. S. presidential election. J. Cog. Neuroscience, 18 (2006), No. 11, 1947-1958.

[56] K.-F. Wong, X.-J. Wang. A recurrent network mechanism of time integration in perceptual decisions. J. Neurosci., 26 (2006), 1314-1328. 


\section{Appendix}

Here is the XPPAUT program used to study the effects of changes in the initial condition and function on the behavior of (4.1). The XPPAUT software can be downloaded without charge from the website http://www.math.pitt.edu/ bard/xpp/xpp.html.

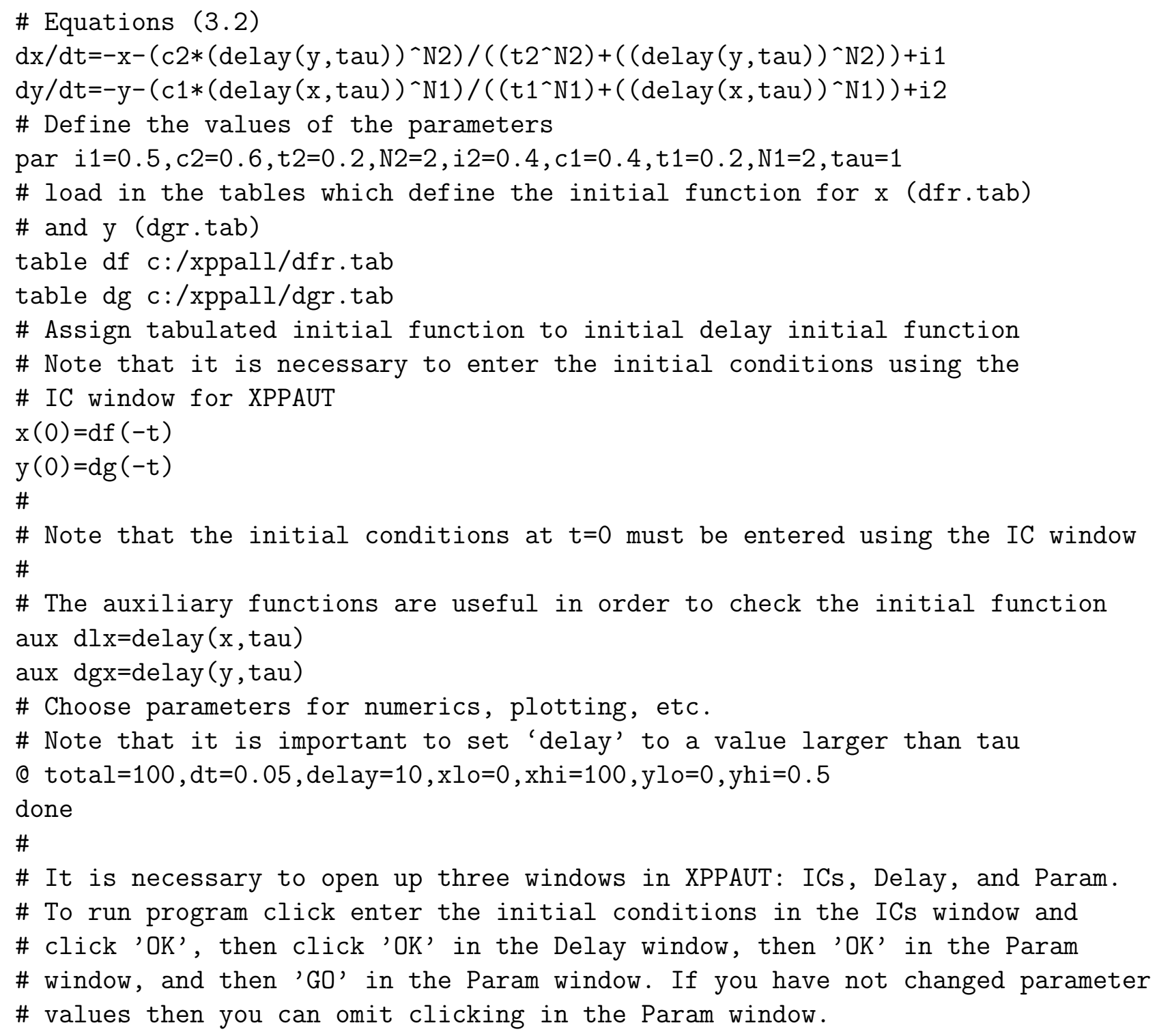

The $x$ and $y$ values for the initial function are stored separately in files names *.tab. The structure of the *.tab files is as follows:

A \# number of pts in the initial function = time delay (tau)/time step

B \# integer smaller that the smallest value in the initial function 
J. Milton et al.

Indecisive decision making

C \# integer greater than the largest value in the initial function

$t-d t$

$t-2 d t$

$\mathrm{t}-\mathrm{tau}$ 